In Cres. Vol. $3 N^{\circ} 1:$ pp. 121-129, 2012

\title{
ESTADO NUTRICIONAL Y RENDIMIENTO ACADÉMICO DEL ESCOLAR*
}

\author{
NUTRITIONAL STATUS AND THE STUDENT'S \\ ACADEMIC ACHIEVEMENT
}

\author{
Maria Elena Urquiaga Alva ${ }^{l}$, \\ Carmen Gorritti Siappo ${ }^{2}$
}

\section{RESUMEN}

El presente trabajo de investigación se realizó con el objetivo de establecer la relación entre el estado nutricional y el rendimiento académico del escolar de la Institución Educativa "República de Chile" de Casma. El diseño de investigación fue descriptivocorrelacional. La población estuvo constituida por 558 escolares y la muestra fue de 228 . Se utilizaron dos instrumentos para la recolección de datos: el Registro de evaluación de los aprendizajes y las tablas de evaluación nutricional. Ambos fueron evaluados en su validez y confiabilidad. Para el procesamiento de datos y los cálculos se hizo uso del programa estadístico SPSS para entorno Windows versión 15.0 y se utilizó la prueba de significancia estadística chí cuadrado para la correlación de variables. Los resultados a los que se llegaron en la presente investigación fueron: la mayoría de escolares de la institución educativa República de Chile presentó estado nutricional normal y el mayor porcentaje presentó rendimiento académico con logro previsto A. No existe relación estadísticamente significativa entre el estado nutricional y el rendimiento académico al aplicar la prueba de independencia de criterios chi cuadrado.

PALABRAS CLAVE: Estado nutricional, Rendimiento académico del escolar.

* Recibido: 20 de enero del 2012; aceptado: 14 de junio del 2012.

1 Magister en Enfermería, Mención: Salud de la Mujer y el Niño. Docente en la Carrera Profesional de Enfermería de la Universidad Católica Los Ángeles de Chimbote.

2 Maestra en Enfermería, Mención: Salud familiar y comunitaria. Directora de la Escuela Profesional de Enfermería. Universidad Católica Los Ángeles de Chimbote. 


\begin{abstract}
This descriptive and correlational research was performed in order to establish the relationship between nutritional status and academic achievement of the República de Chile School's student of Casma city. The population consisted of 558 schoolchildren and the sample was 228. Two instruments were use for data collection: The learning assessment registry and the nutritional assessment tables, both were evaluated for their validity and reliability. For data processing and calculations were made using SPSS for Windows version 15.0 and used the Chi square test of statistical significance. The results of this research were: Most of the República de Chile School had normal nutritional status and the highest percentage reached an expected accomplishment in their academic performance. There is no significant relationship between nutritional status and academic achievement.
\end{abstract}

KEY WORDS: Nutritional status, The students's academic performance.

\title{
I. INTRODUCCIÓN
}

Al niño en edad escolar se le encuentra en todas partes; es quizás el grupo etáreo más omnipresente en diferentes grupos; asimismo el grupo que menor atención recibe por parte del sector salud, que centra mayor atención en el niño menor de 5 años, sin considerar los problemas de maltrato, atraso o deserción escolar, trabajos forzosos, privación afectiva de los padres, mala alimentación. Hay una pobreza común, el niño carece de vigilancia, vive en el abandono; muchos sin oportunidades de acceder a los centros educativos y los que lo hacen carecen de las condiciones básicas como: útiles escolares, lonchera escolar, transporte y luz eléctrica. ${ }^{1}$

El Ministerio de Salud (MINSA) tiene una estrategia para atender la salud del escolar, que consiste en brindar atención integral al escolar dentro del paquete de atención al niño y adolescente. Uno de los aspectos que considera como prioritario el MINSA es el estado nutricional en el ciclo escolar, debido a que es una etapa con ritmo de crecimiento estable. Dentro de sus actividades vigila, a través de las medidas antropométricas, el crecimiento y desarrollo para así poder identificar las tendencias en etapas tempranas de los niños. ${ }^{2}$

En el Perú las estadísticas nacionales nos muestran que el $35 \%$ de los niños de 5 a 12 años presenta algún tipo de desnutrición, ${ }^{3}$ siendo la salud, específicamente el estado nutricional, importante para el rendimiento académico del escolar, debido a que va estar relacionado con la función cognoscitiva y conociendo el reporte estadístico de bajo rendimiento de los escolares en nuestra localidad, me motivó a investigar el "Estado nutricional y su rendimiento académico del escolar". 
Gonzales, ${ }^{4}$ en Cuba, realiza un estudio titulado "La desnutrición y el rendimiento escolar en el nivel primario". En una muestra de 120 alumnos utilizó como instrumento las tablas nutricionales de Waterloo y un examen de conocimientos. Reporta que el $80 \%$ de niños que presentaron desnutrición obtuvieron un bajo rendimiento académico.

Álvarez, ${ }^{5}$ en su estudio realizado en Trujillo, "La Nutrición y su influencia en el rendimiento académico de los niños y las niñas de primer ciclo de Educación Primaria", utilizó una muestra de 220 alumnos y concluye que la nutrición influye en el rendimiento académico de los escolares.

La enfermera, a través del cuidado integral que brinda al niño y al adolescente, considera los índices antropométricos y evalúa el estado nutricional, y a través de la experiencia profesional se ha podido identificar, que un alto porcentaje de escolares presentan alteraciones nutricionales.

\section{PROBLEMA}

¿Existe relación entre el estado nutricional y el rendimiento académico del escolar de 6 a 11 años de la Institución Educativa "República de Chile", de Casma, 2006?

\section{OBJETIVO GENERAL}

Establecer la relación entre el estado nutricional y el rendimiento académico del escolar de 6 a 11 años de la Institución Educativa "República de Chile", de Casma.

\section{OBJETIVOS ESPECÍFICOS}

1. Determinar el rendimiento académico del escolar de 6 a 11 años de la Institución Educativa "República de Chile", de Casma.

2. Determinar el estado nutricional del escolar de 6 a 11 años de la Institución Educativa "República de Chile", de Casma.

El presente trabajo de investigación se sustenta en el modelo de promoción de la salud de Nola Pender y las bases conceptuales sobre aprendizaje y características del escolar y estado nutricional.

Pender, ${ }^{6,7}$ en su modelo de promoción de la salud, afirma que promocionar la salud significa evitar o disminuir factores de riesgo y crear o aumentar los factores protectores, cambiando estilos de vida, creando una cultura de la salud y haciendo de ésta un valor. Pender, al plantear su modelo, nos señala 
que las personas que otorgan gran importancia a su salud, tienen mayor tendencia a conservarla. Asimismo, plantea que las motivaciones personales, creencias y mitos son el motor fundamental que mueve nuestras conductas a favor o en contra de la salud; es así que en el presente trabajo se estudió la relación entre el rendimiento académico y el estado nutricional del niño del niño escolar.

El proceso de aprendizaje, según Piaget, a lo largo del desarrollo la persona irá construyendo, en un proceso de interacción con los objetos, determinadas estructuras o totalidades organizadas en esquemas de acción que obedecen a ciertas "reglas" o "leyes". Las sucesivas estructuras que se van construyendo, suponen formas de relación y comprensión de la realidad cada vez más potentes. "Ningún razonamiento es posible sin experimentar sentimientos". Por lo tanto, el aspecto emotivo, afectivo, valorativo, actitudinal y volitivo tendrá que ser un constitutivo importante de toda propuesta en educación. ${ }^{8}$

El estado nutricional en condiciones normales es la resultante del balance entre lo consumido y lo requerido, lo cual está determinado por la calidad de nutrientes de la dieta y por su utilización completa en el organismo. La valoración nutricional debe formar parte integral de toda evaluación clínica, con el fin de identificar personas que requieren un soporte nutricional agresivo y temprano con el fin de disminuir los riesgos. Una alimentación adecuada, porque los niños y adolescentes, al crecer, requieren de mayor cantidad de proteínas, vitaminas y carbohidratos que los adultos, pues estos nutrientes son los que permiten al organismo ir construyendo los nuevos tejidos que demanda su estatura cada vez mayor. ${ }^{9,10}$

El escolar pasa por un periodo de grandes cambios, de inestabilidad emocional, tiene mayor contacto con situaciones de riesgo, se encuentra en búsqueda de su autonomía personal y de compensación. Asimismo, es una etapa en que está más entregado al juego, descuidando muchas veces la alimentación. También este grupo es dedicado más tiempo al aprendizaje, ocasionando un mayor desgaste de energía, necesitando una mejor nutrición de acuerdo a sus requerimientos nutricionales, que muchas veces no son satisfechas. ${ }^{11}$

\section{METODOLOGÍA}

El presente trabajo constituye una investigación de enfoque cuantitativo de tipo no experimental. El diseño de investigación es Descriptivo-correlacional. Se trata también de descripciones, pero no de categorías, conceptos, objetos ni variables individuales, sino de sus relaciones. ${ }^{12}$ 
La muestra estuvo constituida por 228 alumnos escolares de 6 a 11 años de la Institución Educativa "Republica de Chile", de Casma.

Los conceptos del presente estudio fueron:

Estado nutricional del escolar, definido como la relación entre el peso expresado en kilogramos sobre el cuadrado de la talla expresada en metros, representada por el índice de masa corporal. ${ }^{13}$

Rendimiento académico del escolar. Es el grado de desarrollo o adquisición alcanzado por el estudiante en relación con los aprendizajes previstos o esperados ${ }^{(13)}$. Se medirá en escala ordinal, de acuerdo a las siguientes categorías:

Logro destacado (AD). Cuando el estudiante evidencia el logro de los aprendizajes previstos, demostrando incluso un manejo solvente y satisfactorio en todas las tareas propuestas. ${ }^{14}$

Logro previsto (A). Cuando el estudiante evidencia el logro de los aprendizajes previstos en el tiempo. ${ }^{14}$

En proceso (B). Cuando el estudiante está en camino de lograr los aprendizajes previstos, para lo cual requiere acompañamiento durante un tiempo razonable para lograrlo. ${ }^{14}$

En inicio (C). Cuando el estudiante está empezando a desarrollar los aprendizajes previstos o evidencia dificultades para el desarrollo de éstos, necesitando mayor tiempo de acompañamiento e intervención del docente. ${ }^{14}$

Para la recolección de datos, en el presente trabajo de investigación se solicitó, a través de documento, el permiso del Director de la institución educativa para la evaluación nutricional y la obtención de notas del I semestre. Se obtuvo el consentimiento de los padres, se acordó una fecha de reunión conjunta entre docentes y padres y se programó la evaluación nutricional de los estudiantes de 6 a 11 años.

\section{RESULTADOS Y DISCUSIÓN}

En la tabla 01, sobre rendimiento académico de los escolares de la IE "República de Chile", de Casma 2006, se observa que un 57,46\% de los alumnos de 6 a 11 años, de Primaria, alcanzaron una calificación A (logro previsto) y un 42 , $54 \%$ concluyeron sus estudios con una calificación B (logro en proceso).

En la tabla 02, sobre estado nutricional de los escolares de la IE "República de Chile" de Casma, en el año 2006, de acuerdo a su correspondiente rango percentilar, se observa que un $4,82 \%$ presenta déficit de peso; un $9,65 \%$, bajo peso; el 70,61\%, peso normal; el 9,65\%, sobrepeso y el 5,26\%, obesidad. 
Tabla 01

RENDIMIENTO ACADÉMICO DE LOS ESCOLARES DE LA IE “REPUBLICA DE CHILE”, CASMA, 2006

\begin{tabular}{lcc}
\hline Rendimiento académico & $\mathbf{N}^{\circ}$ & $\mathbf{\%}$ \\
\hline En inicio & 0 & 0,0 \\
Logro Previsto A & 131 & 57,46 \\
Logro En Proceso B & 97 & 42,54 \\
\hline Total & 228 & 100,0 \\
\hline Fuente: Registro de Evaluación de los Aprendizajes. & \\
Elaborado por Urquiaga, M. Casma. 2006. &
\end{tabular}

Tabla 02

ESTADO NUTRICIONAL DE LOS ESCOLARES DE LA IE "REPUBLICA DE CHILE”, CASMA, 2006

\begin{tabular}{lcc}
\hline & $\mathbf{N}^{\circ}$ & $\%$ \\
\hline Déficit de peso & 11 & 4,8 \\
Bajo peso & 22 & 9,6 \\
Normal & 161 & 70,6 \\
Sobrepeso & 22 & 9,6 \\
Obeso & 12 & 5,2 \\
\hline Total & 228 & 100,0 \\
\hline
\end{tabular}

Fuente: Tabla nutricional de índice de masa corporal. Elaborado por MINSA. 2006. Casma, 2006.

En la tabla 03, sobre relación entre el estado nutricional y el rendimiento académico del escolar de Casma, año 2006, se puede observar que del total de alumnos, el $57,5 \%$ ha logrado una calificación A (logro previsto) y un $42,5 \%$ alcanzó B (logro en proceso). El 45,5\% de los alumnos que tienen déficit de peso tienen la calificación de aprendizaje A (logro previsto) y el $54,5 \%$ calificación B (logro en proceso). De los alumnos con peso bajo, el $63,6 \%$ tiene A (logro previsto) y el $36,4 \%$ tiene B (logro en proceso). El $57,8 \%$ de los alumnos que tienen peso normal tienen A (logro previsto) y un $42,2 \%$ tienen B (logro en proceso). De los alumnos que tienen sobrepeso un $63,6 \%$ tienen A (logro previsto) y un $36,4 \%$ tienen B (logro en proceso). De los alumnos que son obesos un 41,7\% tienen A (logro previsto) y un 58,3\% tienen B (logro en proceso). 
Tabla 03

RELACIÓN ENTRE EL ESTADO NUTRICIONAL Y EL RENDIMIENTO ACADÉMICO

\begin{tabular}{llccc}
\hline & & \multicolumn{2}{c}{ Rendimiento } & \\
\cline { 3 - 4 } & & $\begin{array}{c}\text { Logro } \\
\text { previsto A }\end{array}$ & $\begin{array}{c}\text { Logro en } \\
\text { proceso B }\end{array}$ & Total \\
\hline \multirow{2}{*}{ Déficit de peso } & Estado Nutricional & $45,5 \%$ & $54,5 \%$ & $100,0 \%$ \\
& $\%$ del Total & $2,2 \%$ & $2,6 \%$ & $4,8 \%$ \\
\hline \multirow{2}{*}{ Bajo peso } & Estado Nutricional & $63,6 \%$ & $36,4 \%$ & $100,0 \%$ \\
& \% del Total & $6,1 \%$ & $3,5 \%$ & $9,6 \%$ \\
\hline \multirow{2}{*}{ Normal } & Estado Nutricional & $57,8 \%$ & $42,2 \%$ & 100,0 \\
& $\%$ del Total & $40,8 \%$ & $29,8 \%$ & $70,6 \%$ \\
\hline \multirow{2}{*}{ Sobrepeso } & Estado Nutricional & $63,6 \%$ & $36,4 \%$ & 100,0 \\
& $\%$ del Total & $6,1 \%$ & $3,5 \%$ & $9,6 \%$ \\
\hline \multirow{2}{*}{ Total } & Estado Nutricional & $41,7 \%$ & $58,3 \%$ & $100,0 \%$ \\
& $\%$ del Total & $2,2 \%$ & $3,1 \%$ & $5,3 \%$ \\
\hline
\end{tabular}

Fuente: Registro de Evaluación de los Aprendizajes. Elaborado por Urquiaga A. Casma. 2006.

Tabla Nutricional de Índice de masa corporal. Elaborado por MINSA. Casma 2006.

$$
\mathrm{X}^{2}=2,56 \quad \text { GI: } 4 \quad \text { Sig: } 0,63
$$

\section{DISCUSIÓN}

En los resultados de la presente investigación se evidencia que existen diferencias favorables para el grupo de alumnos que alcanzaron una calificación A; sin embargo, casi la mitad alcanzaron una calificación $\mathrm{B}$, lo cual nos indica que el estudiante está en camino de lograr aprendizajes previstos.

Estos hallazgos son similares a los encontrados por Fairchil ${ }^{15}$ y Morillo $^{16}$, quienes señalan que existe un bajo rendimiento académico en gran proporción en escolares con prevalencia mayor del $50 \%(0,67)$. El rendimiento escolar es un aspecto del proceso educativo, en el que influyen múltiples factores causales, así como el nivel socioeconómico bajo, características de la familia de los alumnos y de su comunidad, habilidades innatas de los alumnos, inasistencia, maltrato infantil, etc. ${ }^{14}$

Esta conjunción de factores negativos conspiran contra el desempeño escolar de los niños. Fairchil ${ }^{15}$ y Morillo ${ }^{16}$ demostraron que casi un cuarto de los niños al ingresar al primer grado de la escuela tienen un coeficiente intelectual 
inferior a lo esperable. Uno de los factores responsables de este bajo desempeño es la inadecuada estimulación temprana por parte de progenitores que ignoraban como hacerlo. ${ }^{17}$

Se evidencia que existe un porcentaje significativo de estudiantes con peso por debajo de lo normal $(14,4 \%)$. Estos hallazgos son similares a un estudio encontrado en Lima, donde se evidencia que un $30 \%$ de escolares presenta un peso por debajo de lo normal y aunado a esto, baja hemoglobina. En otro trabajo de investigación aplicado en Huancavelica en una Institución Educativa, se encontró que la tercera parte de escolares presentó deficiencias nutricionales, ocasionando en ellos la deserción escolares y un bajo rendimiento. ${ }^{18}$

La malnutrición es uno de los problemas que más afecta a la población infantil de los países en vías de desarrollo. El término "malnutrición" engloba estados nutricionales, como la desnutrición y la obesidad. Un estado de nutrición óptima asegura a un niño con menos problemas de salud y con más facilidades en el aprendizaje. En las instituciones educativas del Perú se adolece de una buena estrategia nutritiva para los educandos, hecho que conlleva a que los educandos no tengan un adecuado rendimiento académico. Esto se debe a la falta de una información adecuada sobre los nutrientes alimenticios. ${ }^{19}$

De acuerdo al estadístico de Prueba $\mathrm{X}^{2}(2,56)$ y su valor $\mathrm{sig}=0,63$, se concluye que no existe una relación significativa entre las dos variables en estudio, por lo que si aceptamos lo contrario, siendo falso, nos estaríamos equivocando en un $63,0 \%$, de tal modo que no podemos rechazar la hipótesis nula y aceptar que ambas variables son dependientes o están asociadas o relacionadas.

Debido a que la relación o asociación entre el estado nutricional de los alumnos de la I.E. "República de Chile", de Casma, no es significativa, se concluye que ambas no están asociadas o correlacionadas. Es decir, no existe relación significativa entre el estado nutricional y el rendimiento académico, pero existen otros factores que probablemente influyen en el rendimiento académico, así como desintegración familiar, baja autoestima, problemas de drogadicción, maltrato infantil, deserción escolar y desnutrición.

Esto se afirma con otros hallazgos encontrados, como el que fue aplicado en la ciudad de Lima, donde un grupo de escolares presentan un bajo rendimiento por presentar maltrato infantil. Asimismo, en un estudio demostraron que casi un cuarto de los niños al ingresar al primer grado de la escuela tiene un coeficiente intelectual inferior a lo esperable. Uno de los factores responsables de este bajo desempeño es la inadecuada estimulación temprana por parte de progenitores. ${ }^{20}$ 


\section{CONCLUSIONES}

1. El mayor porcentaje de escolares ha alcanzado en su rendimiento académico un logro previsto $\mathrm{A}$.

2. La mayoría de los escolares tienen un estado nutricional normal, seguido de escolares con sobrepeso y bajo peso.

3. No existe relación estadísticamente significativa entre el estado nutricional y el rendimiento académico.

\section{REFERENCIAS BIBLIOGRÁFICAS}

1 Anguera M. Metodología de la observación en las mediciones. Madrid. Cátedra, 1989.

2 BABBIE E. Manual para la práctica de la investigación social. Bilbao. Desclée de Brouwer, 1989.

3 Colegio de Nutricionistas del Perú. Nutrición y Salud. Benavente Iyaray, Niveles de Pobreza, Modelos de Ajuste y repercusión en la Nutrición. Perú, 1997.

4 Ministerio de EduCACión. Población escolar del Perú. Perú, 2002.

5 Hidalgo P. "Estado nutricional en escolares en escolares de 6 a 12 años de edad. Trujillo, 2001.

6 Colegio de Nutricionistas Del Perú. X Congreso Latinoamericano de Nutricionistas y Dietistas. Servigraf .América S.L. Lima-Perú, 1995.

7 Del Rey C y Calvo, F. Como Cuidar la Salud, Su Educación y Promoción. España, editorial Harcout Brice, 1998.

8 HERnÁNDEZ R. Metodología de la Investigación. México, Editorial Mc Graw-Hill Interamericana, 1997.

9 MARINE. Estado nutricional en el escolar. México, Editorial Interamericana, 1999.

10 Marriner, T. Modelos y Teorías en enfermería. $3^{\circ}$ edición. España ,Editorial Harcout Brice, 1994.

11 Piaget J. Psicología y pedagogía. Barcelona, 2002.

12 CAstillo C. “ La Lonchera Nutritiva y su Influencia con el Rendimiento Académico de los Niños y las Niñas de Primer Ciclo de Educación Primaria”. Chimbote, 2003.

13 Campbell D. Y Stanley J. Diseños experimentales y cuasiexperimentales en la investigación social. Buenos Aires, 2002.

14 Denise F. Polit, D. Investigación científica en ciencias de la Salud. México, McGrawHillInteramericana. 1990.

15 MoISÉs H. “Estado nutricional en Escolares”. Perú, 2001.

16 Ministerio de SAlud. Manual para la Educación en Salud Integral del Escolar y Adolescente. Subprograma Nacional Salud Escolar Adolescente. Perú, 1997.

17 Avolio D. Planeamiento del Proceso de Enseñanza Aprendizaje. 4ta Edición. Editorial Marymar. Lima-Perú, 1994.

18 Mosby. Tratado de Enfermería. México, Editorial. Océano, 1996.

19 FRANCISCO H. "Estado nutricional en escolares”. Trujillo-Perú, 2003.

20 CRUTER N. Compendio de Nutrición. $3^{\circ}$ Edición. México, Editorial Iberamericana, 2002. 
